Escherichia coli beta-galactosidase is known to be active only in the form of tetramers, while the cold-active Arthrobacter sp. C2-2 beta-galactosidase forms compact hexamers with active sites oriented into an internal cavity, connected by three types of channels with exterior solvent. Additionally, sequence differences between both enzymes exist in the active site.

Acknowledgement: The research was supported by the Grant Agency of the Czech Republic (project 204/02/0843/A) and by the Grant Agency of the Academy of Sciences of the Czech Republic (project B500500512).

[1] Skalova T, Dohnalek J, Spiwok V, Lipovova P, Vondrackova E, Petrokova H, Duskova J, Strnad H, Kralova B, Hasek J, J. Mol. Biol., 2005, 353(2), 282.

\section{MS03 P21}

Pyrroloquinoline Quinone (PQQ) Biosynthesis Sandra Puehringer and Robert Schwarzenbacher Structural Biology, University of Salzburg, Billrothstr 11, 5020 Salzburg, Austria. E-mail: sandra.puehringer@sbg.ac.at

The biosynthesis of pyrroloquinoline quinone (PQQ), a novel vitamin and redox cofactor of quinoprotein dehydrogenases, is facilitated by an unknown pathway that requires the expression of six genes (pqqA-F) to derive PQQ from glutamate and tyrosine residues encoded in the precursor peptide PqqA. In previous work we successfully characterized the last step of PQQ biosynthesis and discovered that PqqC (EC 1.3.3.11) is a novel cofactorless oxygenase (PNAS 2004; JACS 2004; Proteins 2004). PqqC catalyzes the final step in the pathway in a reaction that involves ring cyclization and eight electron oxidation of 3a-(2-amino-2-carboxy-ethyl)-4,5-dioxo-4,5,6,7,8,9hexahydro-quinoline-7,9-dicarboxylic-acid to PQQ. Here, we describe the crystal structures of $\mathrm{PqqC}$ and its complex with PQQ and determine the stoichiometry of $\mathrm{H}_{2} \mathrm{O}_{2}$ formation and $\mathrm{O}_{2}$ uptake during the reaction. The $\mathrm{PqqC}$ structure reveals a compact seven-helix bundle that provides the scaffold for a positively charged active site cavity. Product binding induces a large conformational change, which results in the active site recruitment of amino acid side chains proposed to play key roles in the catalytic mechanism. PqqC is unusual in that it transfers redox equivalents to molecular oxygen without the assistance of a redox active metal or cofactor. The structure of the enzyme-product complex shows additional electron density next to R179 and C5 of PQQ, which can be modeled as $\mathrm{O}_{2}$ or $\mathrm{H}_{2} \mathrm{O}_{2}$, indicating a site for oxygen binding. We propose a novel reaction sequence that involves base-catalyzed cyclization and a series of quinone-quinol tautomerizations that are followed by cycles of $\mathrm{O}_{2} / \mathrm{H}_{2} \mathrm{O}_{2}$-mediated oxidations.

\section{MS03 P22}

Structural studies of the dual-substrate TIM-barrel phosphoribosyl isomerase A Helena Wright ${ }^{\mathrm{a}}$, Lianet Noda-García ${ }^{\mathrm{b}}$, Adrián Ochoa-Leyva ${ }^{\mathrm{b}}$, David A Hodgson ${ }^{\mathrm{a}}$, Francisco Barona-Gómez ${ }^{\mathrm{a}, \mathrm{b}}$ and Vilmos Fülöp ${ }^{\text {a }}{ }^{\mathrm{a}}$ Department of Biological Sciences, University of Warwick, $U K{ }^{\mathrm{b}}{ }^{\mathrm{b}}$ Departamento de Ingeniería Celular $y$ Biocatálisis, Instituto de Biotecnología, Universidad Nacional Autónoma de México (UNAM).

E-mail: Helena.wright@,warwick.ac.uk

Keywords: $(\beta \alpha)_{8}$-barrel, histidine and tryptophan biosynthsesis, evolution.

Phosphoribosyl isomerase A (PriA) is a (betaalpha) $)_{8}$ TIM barrel enzyme involved in both histidine (HisA activity) and tryptophan (TrpF activity) biosynthesis [1]. Despite the availability of a 3D structure of PriA, its structure / function relationship remains poorly defined. Since PriA has ancient-like features, i.e. a dual-substrate specificity within a physiological context, this (betaalpha) ${ }_{8}$-barrel represents a suitable model system to explore enzyme function evolutionary hypotheses. Thus, elucidating the structure / function relationship of this enzyme may have a bearing on the evolution of the (betaalpha) ${ }_{8}$-fold as a whole. Here, we will discuss a novel crystallographic structure of PriA resolved at $1.8 \AA$, which shows the regions that appear disordered in a previously reported structure (PDB: 1vzw). Based on these structural differences, the function of this novel (betaalpha) ${ }_{8}$-barrel enzyme has been predicted. These predictions, have been validated through mutagenesis and functional analysis.

[1] Barona-Gómez, F., Hodgson, D.A., EMBO Rep., 2003,4,296 